\title{
Towards Accurate and Label-free Monitoring of Bio-analytes using Supercontinuum based Multispectral Photoacoustic Spectroscopy in the Extended Near-infrared Wavelength regime
}

Dasa, Manoj Kumar; Markos, Christos; Janting, Jakob; Adamu, Abubakar Isa; Bowen, Patrick ; Bang, Ole

Published in:

Proceedings of SPIE

Link to article, DOI:

$10.1117 / 12.2510284$

Publication date:

2019

Document Version

Publisher's PDF, also known as Version of record

Link back to DTU Orbit

Citation (APA):

Dasa, M. K., Markos, C., Janting, J., Adamu, A. I., Bowen, P., \& Bang, O. (2019). Towards Accurate and Labelfree Monitoring of Bio-analytes using Supercontinuum based Multispectral Photoacoustic Spectroscopy in the Extended Near-infrared Wavelength regime. In Proceedings of SPIE (Vol. 10890). [108900W] SPIE International Society for Optical Engineering. Proceedings of SPIE - The International Society for Optical Engineering https://doi.org/10.1117/12.2510284

\section{General rights}

Copyright and moral rights for the publications made accessible in the public portal are retained by the authors and/or other copyright owners and it is a condition of accessing publications that users recognise and abide by the legal requirements associated with these rights.

- Users may download and print one copy of any publication from the public portal for the purpose of private study or research.

- You may not further distribute the material or use it for any profit-making activity or commercial gain

- You may freely distribute the URL identifying the publication in the public portal 


\section{PROCEEDINGS OF SPIE}

\section{Towards accurate and label-free monitoring of bio-analytes using supercontinuum based multispectral photoacoustic spectroscopy in the extended near-infrared wavelength regime}

Manoj Kumar Dasa, Christos Markos, Jakob Janting, Abubakar I. Adamu, Patrick Bowen, et al.

Manoj Kumar Dasa, Christos Markos, Jakob Janting, Abubakar I. Adamu, Patrick Bowen, Ole Bang, "Towards accurate and label-free monitoring of bio-analytes using supercontinuum based multispectral photoacoustic spectroscopy in the extended near-infrared wavelength regime," Proc. SPIE 10890, Label-free Biomedical Imaging and Sensing (LBIS) 2019, 108900W (4 March 2019); doi: 10.1117/12.2510284

SPIE. Event: SPIE BiOS, 2019, San Francisco, California, United States 


\title{
Towards Accurate and Label-free Monitoring of Bio-analytes using Supercontinuum based Multispectral Photoacoustic Spectroscopy in the Extended Near-infrared Wavelength regime
}

\author{
Manoj Kumar Dasa ${ }^{*}$, Christos Markos ${ }^{\text {a }}$, Jakob Janting ${ }^{\text {a }}$, Abubakar I. Adamu ${ }^{\text {a }}$, Patrick \\ Bowen $^{\mathrm{b}}$, and Ole Bang ${ }^{\mathrm{a}, \mathrm{b}}$ \\ ${ }^{a}$ DTU Fotonik, Department of Photonics Engineering, Technical University of \\ Denmark, Kgs. Lyngby 2800, Denmark \\ ${ }^{\mathrm{b}}$ NKT Photonics, Blokken 84, Birkerød 3460, Denmark \\ *manda@fotonik.dtu.dk
}

\begin{abstract}
In this work, we report a cost-effective supercontinuum (SC) laser-based multispectral photoacoustic spectroscopy (MSPAS) system for studying spectral characteristics of various analytes. We demonstrate an in vitro label-free monitoring of the analytes in the extended near-infrared (NIR) (1540-1840 nm) spectral range. We further demonstrate how a simple ratiometric analysis in conjunction with linear regression can be used for accurate prediction of glucose over commonly encountered physiological levels inside the human body $(0-400 \mathrm{mg} / \mathrm{dL})$. Looking forward, the proposed SC-based MSPAS system provides a framework for the development of label-free and non-invasive monitoring multiple bio-analytes accurately, with potential translation to clinical in vivo applications.
\end{abstract}

Keywords: Multispectral photoacoustic spectroscopy, supercontinuum lasers, glucose monitoring

\section{INTRODUCTION:}

Accurate monitoring of bio-analytes inside the human body is of great interest for identification and diagnosis of various diseases. Glucose is one such bio-analytes, which is highly coveted for the interrogation of a fatal metabolic disorder known as Diabetes Mellitus (DM). An improper interrogation of DM would lead to the onset of severe chronic complications inside the human body ${ }^{1}$. Hence, several attempts are being made for devising reliable non-invasive and label-free techniques for the monitoring of glucose levels ${ }^{2-4}$. Photoacoustic spectroscopy (PAS) is capable of providing label-free information of the sample based on its endogenous contrast. PAS has attracted significant attention recently for studying various bio-analytes. Specifically, the mid-infrared region was widely employed for glucose detection due to the presence of fundamental absorption bands ${ }^{5-8}$ (9-11 um). However, the strong water absorption within this spectral region imposes significant limitations for non-invasive glucose measurements. Extended-NIR region provides promising abilities for devising label-free and non-invasive techniques for monitoring glucose, due to the presence of overtone and combination bands of $\mathrm{C}-\mathrm{H}$ and $\mathrm{O}-\mathrm{H}$ molecules which are present inside the glucose molecules. Therefore, established non-invasive monitoring techniques are mostly based on commercially available monochromatic laser systems in extended- NIR ${ }^{9-11}$. However, since the absorption of glucose has similar characteristics to that of other bio-analytes (like water and

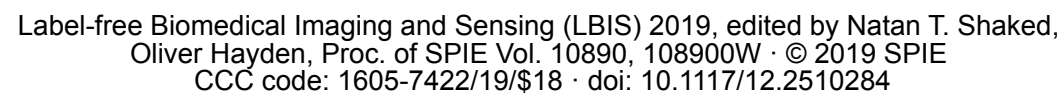


cholesterol), the monochromatic based monitoring techniques have limited sensitivity ${ }^{12-16}$. SC lasers offer an attractive all-fiber alternative to generate broadband emission from ultra violet (UV) to mid infrared region (MIR) of electromagnetic spectrum ${ }^{17-19}$. For this goal, we present a method to accurately measure glucose concentrations in vitro using an SC-based MSPAS system. The proposed method is based on a two-step approach; initially, the spectral characteristics of the bio-analytes are established in the complete wavelength region of interest using a home-built tunable laser system $(1540-1840 \mathrm{~nm})$. We then apply a ratiometric analysis at the wavelength, where glucose shows higher absorption contrast with respect to the other bio-analytes, thereby enabling accurate concentrations.

\section{EXPERIMENTAL SETUP:}

Figure 1 shows a schematic of the MSPAS system used for both the characterization of glucose spectra and the glucose concentration experiments. The system employs a home-built SC laser in conjunction with a linear variable filter (LVF) as the tunable excitation source. The output from the laser can be tuned from 1500-1900 nm. However, the bandwidth of the filtered light is limited to the LVF (2\% of the excitation wavelength). The filtered light from the source is collimated using an aspheric lens (L1), steered using a mirror (M1) and then focused into the sample solution (about 5 $\mathrm{mm}$ above the surface of the sample holder) using an objective lens (L2).

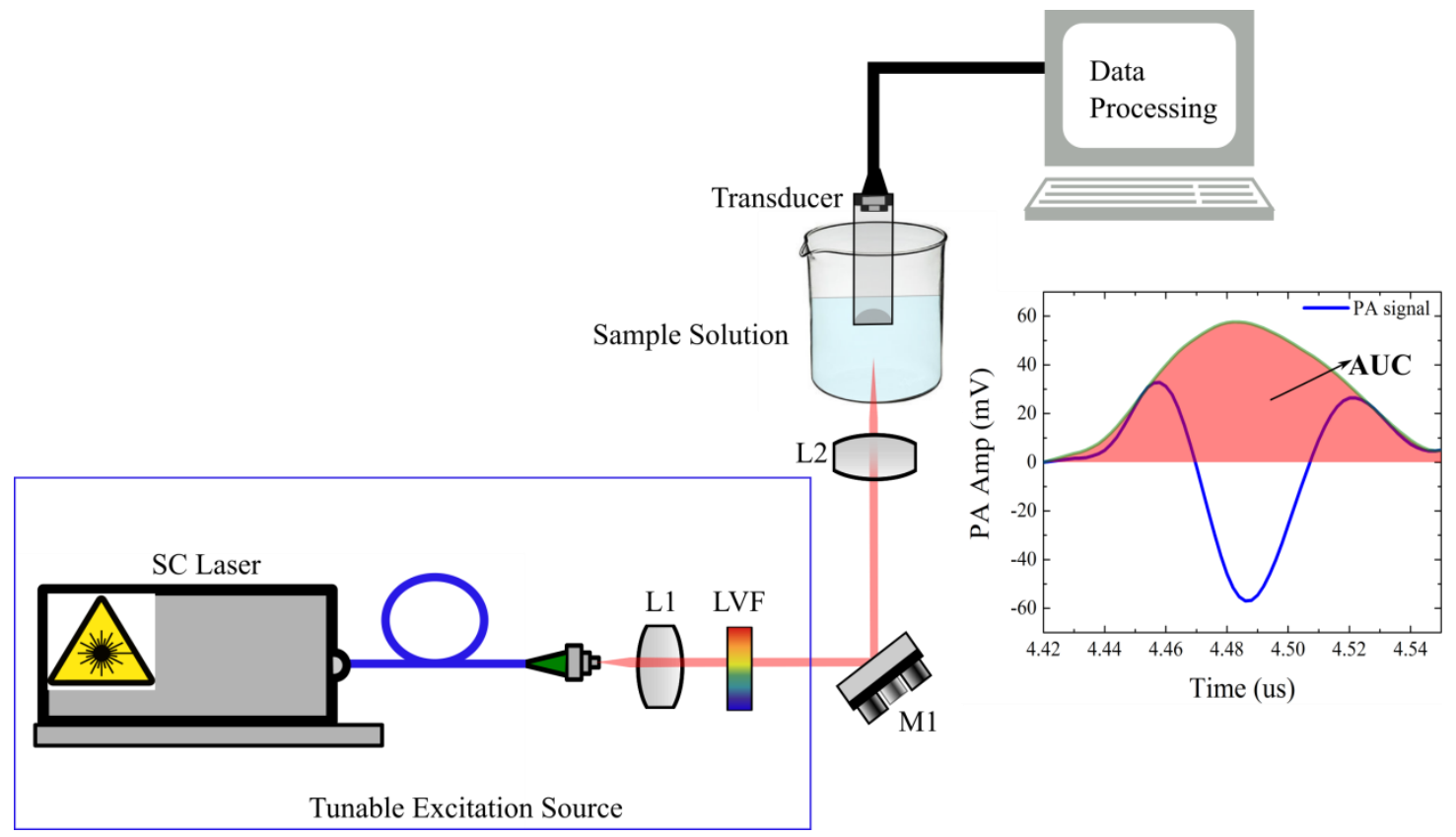

Figure 1: Schematic of the MSPAS system. A home-built SC laser in conjunction with the LVF is used as optical excitation source; the output from the laser is collimated using a lens (L1) and then focused inside the sample solution using an objective lens (L2). The generated PA signals are detected using a focused transducer, amplified using a low-noise amplifier then sent for further data processing. The inset shows the recorded PA signal and its computed envelope signal. 
The generated PA signal is detected using a commercial 7.5 MHz ultrasonic focused transducer. The detected signals are then filtered by a low pass filter, amplified using two cascaded low-noise wideband signal amplifiers and are then further processed. The samples of glucose solutions were prepared by dissolving different proportions of glucose weighed using a sensitive balance (0-400 mg in steps of $50 \mathrm{mg}$ ) and are then dissolved in the distilled water.

\section{RESULTS AND DISCUSSIONS:}

In order to accurately monitor a bio-analyte like glucose using PAS, it is very important to first establish its absorption characteristics, as the PA amplitude of the analyte depends on its absorption properties. To establish the absorption characteristics of the glucose in the extended-NIR, the PA amplitudes of the glucose were recorded from 1540-1840 nm in steps of $20 \mathrm{~nm}$.
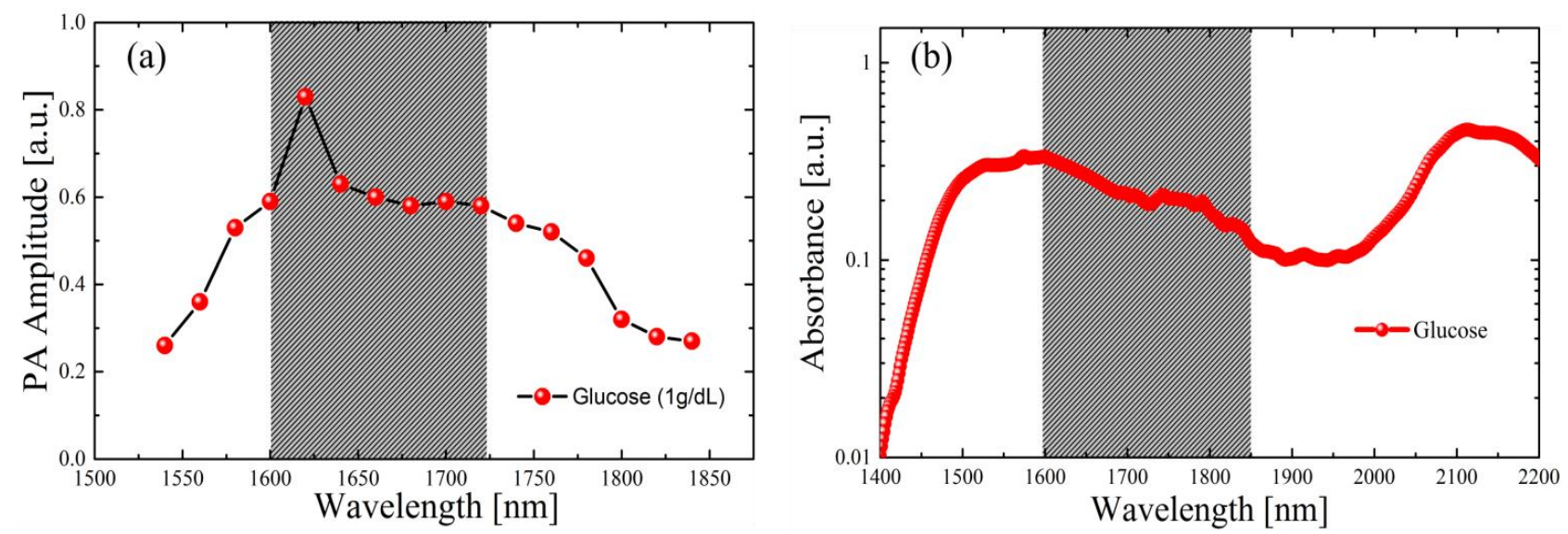

Figure 2(a): Photoacoustic spectra of the glucose solution (1g/dL) measured using the MSPAS system. Figure 2(b): Absorption spectrum of the D-glucose measured using FTIR spectrometer. Inset (highlighted region) shows the first overtone and combination region in the extended-NIR.

Figure 2(a) shows the recorded PA amplitudes as a function of excitation wavelength. The PA amplitude of the recorded PA signal is the computed area under the envelope of the recorded PA signal. Moreover, since the samples used in the experiments were aqueous glucose solutions. A ratiometric analysis was employed to extract the PA amplitude of each concentration with respect to the background (distilled water).

The PA amplitude at a known concentration is given by,

$$
\text { PA amplitude }=\frac{\text { Area under the curve of } P \text { A signal at the known concentration }}{\text { Area under the curve of reference } P \text { A signal (background })}
$$

The PA spectrum shows a peak at $1620 \mathrm{~nm}$, which can be attributed to the increased absorption due to the overtone and combination band in the extended-NIR. Similar absorption characteristics were revealed from the measurements of commercial Fourier transform infrared (FTIR) spectrometer. Figure 2(b) is the absorption spectrum of D-glucose measured using FTIR system. To show the capability of the MSPAS system for label-free bio-analyte monitoring applications, the PA amplitudes of different glucose concentrations varying from 0-400 mg/dL (physiological glucose concentrations inside the human body) in steps of $50 \mathrm{mg} / \mathrm{dL}$ were recorded $(0 \mathrm{mg} / \mathrm{dL}$ is pure distilled 
water). Figure 3 presents the variation of PA amplitude with respect to the concentration of the glucose in the test solution. It can be clearly observed that the PA amplitude variation shows a close to linear correlation, as the increase in the concentration of glucose in the test solution increases the overall absorption, thereby increasing the detected PA amplitude. The computed coefficient of determination $\left(\mathrm{R}^{2}\right)$ of the variation is found to be 0.938 .

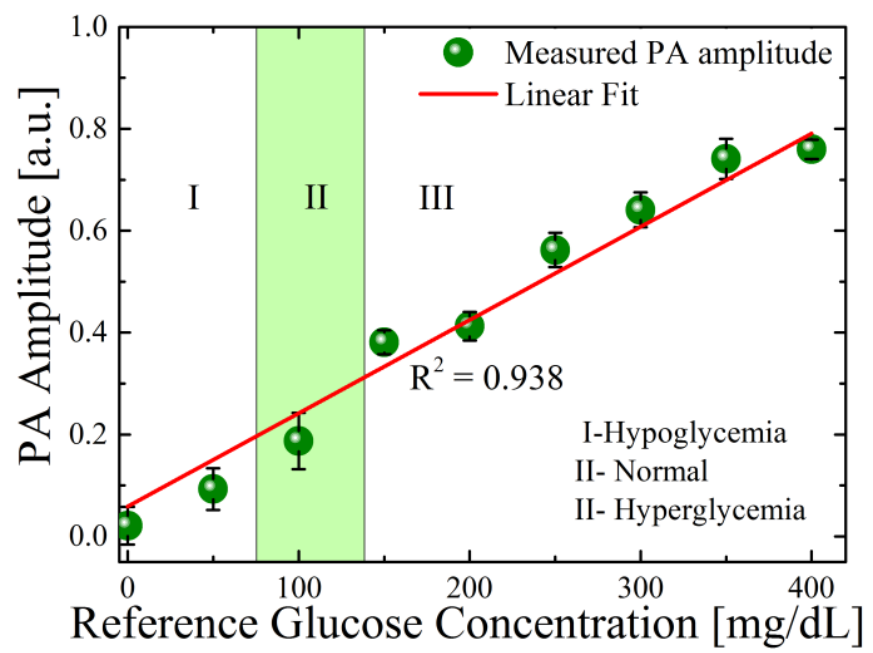

Figure 3: Variation of the PA amplitude as a function of the reference glucose concentration over the commonly encountered physiological concentrations (including regions of hypoglycemia, both normal and hyperglycemia levels) inside the human body.

To further explore the potential of the current MSPAS system for clinical applications, 22 random concentrations of test glucose solutions were prepared and their PA amplitudes were recorded so as to predict the concentration of glucose inside the test glucose solution. The detailed background about the prediction of glucose is described in our previous study ${ }^{16}$. Figure 4 shows the variation of predicted glucose concentration as a function of the reference glucose concentration for 22 random test solutions, ranging over the commonly encountered physiological concentrations inside the human body.

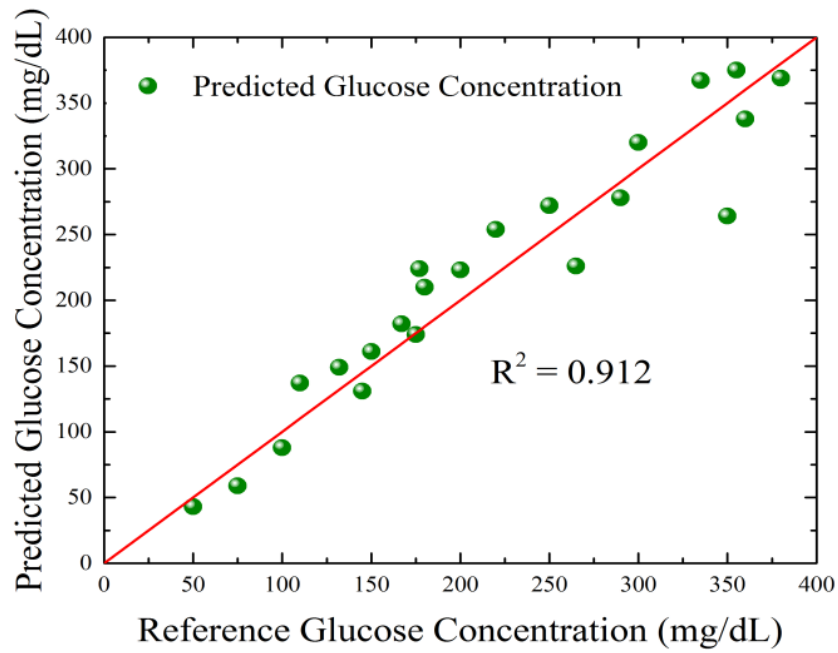

Figure 4: Variation of predicted glucose concentration as a function of the reference glucose concentration of 22 random test solutions, ranging over the commonly encountered physiological concentrations. 
A linear regression ${ }^{16}$ applied to the predicted data set yields a coefficient of determination $\left(\mathrm{R}^{2}\right)$ of 0.912. Thus showcasing the potential of the current system towards accurate and label-free monitoring applications.

\section{CONCLUSION:}

In summary, we reported the development of the MSPAS system based on a home-built SC laser. We then employed the MSPAS system for identifying the absorption characteristics of glucose. We demonstrated how the proposed system can be used to measure glucose concentration using ratiometric analysis over physiological concentrations commonly encountered inside the human body. Using linear regression analysis we further demonstrated that glucose concentrations can be determined for clinical applications with sufficient accuracy (coefficient of determination of 0.912).

\section{ACKNOWLEDGMENTS:}

The research project leading to this work has received funding from the European Union's Horizon 2020 research and innovation programme under the Marie Sklodowska-Curie grant agreement No. 722380. C.M. acknowledges the financial support from the Danish Council for Independent Research (4184-00359B) (8022-00091A). The authors thank Iuliana-Madalina Stoica (University of Copenhagen) for the FTIR measurements and Katharina Haase (University of Heidelberg) for helpful suggestions and discussions.

\section{REFERENCES:}

[1]. The Diabetes Control and Complications Trial Research Group, "The effect of intensified treatment of diabetes on the development and progression of long-term complications in insulindependent diabetes mellitus," N. Engl. J. Med. 329, 977-986 (1993).

[2]. C. Markos, W. Yuan, K. Vlachos, G. E. Town, and O. Bang, "Label-free biosensing with high sensitivity in dual-core microstructured polymer optical fibers," Opt. Express 19, 7790-7798 (2011). [3]. H. U. Hassan, K. Nielsen, S. Aasmul, and O. Bang, "Polymer optical fiber compound parabolic concentrator tip for enhanced coupling efficiency for fluorescence-based glucose sensors," Biomed. Opt. Express 6, 5008-5020 (2015).

[4]. C. Markos and O. Bang, "Nonlinear label-free biosensing with high sensitivity using As2S3 chalcogenide tapered fiber," J. Lightwave Technol. 33(13), 2892-2898 (2015).

[5]. A. Rosencwaig, "Photoacoustic spectroscopy," Annu. Rev. Biophys. Bioeng. 9, 31-54 (1980).

[6]. C. K. N. Patel and A. C. Tam, "Pulsed optoacoustic spectroscopy of condensed matter," Rev. Mod. Phys. 53, 517-550 (1981).

[7]. K. M. Quan, G. B. Christison, H. A. MacKenzie, and P. Hodgson, "Glucose determination by a pulsed photoacoustic technique: an experimental study using a gelatin-based tissue phantom," Phys. Med. Biol. 38, 1911-1922 (1993).

[8]. G. B. Christison and H. A. MacKenzie, "Laser photoacoustic determination of physiological glucose concentrations in human whole blood," Med. Biol. Eng. Comput. 31, 284-290 (1993). 
[9]. J. Y. Sim, C. G. Ahn, E. J. Jeong, and B. K. Kim, "In vivo microscopic photoacoustic spectroscopy for non-invasive glucose monitoring invulnerable to skin secretion products," Sci. Rep. 8, 1059 (2018).

[10]. P. P. Pai, P. K. Sanki, A. De, and S. Banerjee, "NIR photoacoustic spectroscopy for noninvasive glucose measurement," in 37th Annual International Conference of the IEEE Engineering in Medicine and Biology Society (EMBC), pp. 7978-7981 (2015).

[11]. S. Zhao, W. Tao, Q. He, H. Zhao, and W. Cao, "A non-invasive photoacoustic and ultrasonic method for the measurement of glucose solution concentration," AIP Adv. 7, 035313 (2017).

[12]. M. K. Dasa, C. Markos, M. Maria, I. B. Gonzalo, C. R. Petersen, P. M. Moselund, and O. Bang, "Supercontinuum Laser for Spectroscopic Photoacoustic Imaging of Lipids in the Extended Near-Infrared Region," in Frontiers in Optics/Laser Science, OSA Technical Digest (Optical Society of America, 2018), paper FM3F.5.

[13]. M. K. Dasa, C. Markos, M. Maria, C. R. Petersen, P. M. Moselund, and O. Bang, "High-pulse energy supercontinuum laser for high-resolution spectroscopic photoacoustic imaging of lipids in the 1650-1850 nm region," Biomed. Opt. Express 9, 1762-1770 (2018).

[14]. R. Zhang, F. Gao, X. Feng, S. Liu, R. Kishor, Y. Luo, and Y. Zheng, "Noninvasive photoacoustic measurement of glucose by data fusion," Analyst 142, 2892-2896 (2017).

[15]. A. Ghazaryan, S. Ovsepian, and V. Ntziachristos, "Extended near-infrared optoacoustic spectrometry for sensing physiological concentrations of glucose," Front. Endocrinol. 9, 112 (2018). [16]. M K. Dasa, C. Markos, J. Janting and O. Bang, "Multispectral photoacoustic sensing for accurate glucose monitoring using a supercontinuum laser", JOSA B. 36(2), A61-A65 (2019).

[17]. C. R. Petersen, R. D. Engelsholm, C. Markos, L. Brilland, C. Caillaud, J. Trolès, and O. Bang, "Increased mid-infrared supercontinuum bandwidth and average power by tapering large-mode-area chalcogenide photonic crystal fibers," Opt. Express 25(13), 15336-15348 (2017).

[18]. C. Markos, J. C. Travers, A. Abdolvand, B. J. Eggleton, and O. Bang, "Hybrid photonic-crystal fiber," Rev. Mod. Phys. 89(4), 045003 (2017).

[19]. A. I. Adamu, M. S. Habib, C. R. Petersen, B. Zhou, A. Schülzgen, J. E. Antonio-Lopez, R.

Amezcua-Correa, O. Bang, and C. Markos, "Supercontinuum generation from deep-UV to mid-IR in a noble gas-filled fiber pumped with ultrashort mid-IR pulses," in Advanced Photonics 2018 (BGPP, IPR, NP, NOMA, Sensors, Networks, SPPCom, SOF), OSA Technical Digest (online) (Optical Society of America, 2018), paper JTu6E.2. 